\title{
Backscattered Electron Images, X-ray Maps and Monte Carlo simulations applied to the study of plagioclase composition in volcanic rocks
}

\author{
V. Galván Josaa,b,*, D. Fracchiac ${ }^{\mathrm{c}}$, G. Castellano ${ }^{\mathrm{a}, \mathrm{b}}$, E. Crespo ${ }^{\mathrm{d}}$, A. Kang ${ }^{\mathrm{e}}$, \\ R. Bonetto ${ }^{\mathrm{b}, \mathrm{f}}$ \\ ${ }^{a}$ FaMAF, Universidad Nacional de Córdoba, Medina Allende s/n, Ciudad Universitaria, \\ (5016) Córdoba, Argentina. \\ ${ }^{b}$ Consejo Nacional de Investigaciones Científicas y Técnicas (CONICET), Argentina. \\ ${ }^{c}$ Comisión Nacional de Energía Atómica, Avenida del Libertador 8250 (1429), Buenos \\ Aires, Argentina. \\ ${ }^{d}$ Universidad Nacional de San Luis, Argentina. \\ ${ }^{e}$ Laboratorio de Investigaciones de Metalurgia Física (LIMF), Facultad de Ingeniería, \\ Universidad Nacional de La Plata, calle 1 y 47, (1900) La Plata, Argentina. \\ ${ }^{f}$ Centro de Investigación y Desarrollo en Ciencias Aplicadas Dr. Jorge Ronco \\ (CINDECA). Facultad de Ciencias Exactas, Universidad Nacional de La Plata, calle 47 \\ \#257, (1900) La Plata, Argentina.
}

\begin{abstract}
Zoning patterns in plagioclases are related to abrupt changes in the anorthite content along the crystal growing direction. Accurate characterization of these patterns by electron microprobe is useful to identify magma chamber processes such as recharge, mingling and whole-chamber overturn events.

In this work, a new procedure to obtain high resolution quantitative maps of anorthite concentration in single plagioclase crystals is developed. The methodology consists in performing a calibration of backscattered electron images using quantitative X-ray maps. The ultimate resolution of characteristic X-rays and backscattered electron signals is studied by Monte Carlo simulation. The method is applied to characterize the chemical composition of a volcanic plagioclase from the Cerro Vilama, Argentina. The results obtained are more precise than the values given by the methods commonly used in the study of plagioclase composition, i.e. the classical profiling by elec-
\end{abstract}

\footnotetext{
${ }^{*}$ Corresponding author. Tel.: +54 351 4334051; fax +54 3514334054

Email address: galvanvictor@gmail.com (V. Galván Josa)
} 
tron microprobe point analysis or the modern backscattered electron image calibration by means of quantitative energy or wavelength dispersive X-ray analysis at a few selected points.

Keywords:

Backscattered electron imaging, X-ray maps, Plagioclase zoning

\section{Introduction}

The spatial distributions of the components or phases within heterogeneous materials samples is an important issue in a number of research areas. X-ray maps (XRMs) obtained by collecting characteristic X-rays from induced radiative transitions of the elements present in a sample, are particularly helpful for gathering elemental distribution. In the case of electron probes, the beam is scanned across the specimen or the sample is moved under a fixed beam, while X-ray intensities are recorded pixel by pixel. The spatial resolution of XRMs is limited by the interaction volume, which is around $2 \mu \mathrm{m}$ diameter, depending on the electron beam energy, the overall sample composition and the particular element being mapped [1]. The acquisition time required for registering an XRM with $256 \times 256$ pixels is 30 minutes (at $60 \mathrm{kcps}$ ), if relative statistical uncertainties below $2 \%$ are expected. Recent advances in energy dispersive spectrometry have resulted in detection systems with higher counting rates. Silicon drift detectors [2], for example, can produce high quality images at $60 \mathrm{kcps}$ in a few minutes [3].

Backscattered electron (BE) images are also useful to determine the number and distribution of phases, since the gray level of each pixel is related to the mean atomic number $\bar{Z}$ at the corresponding position on the sample surface $[4,5]$, although they cannot be used alone to determine the chemical composition. BE images are quite valuable in different areas of materials characterization, their importance lying in the fact that they are widely used in a variety of scientific or technological investigations, like chemical geology, cement investigations, art and cultural heritage pieces, hard tissue characterization, etc. [6-11].

The plagioclases are a typical and very abundant group of minerals in the igneous rocks of the Earth crust and of the Moon. They form a continuous isomorphic series (solid solution) varying compositionally from a sodic extreme (called albite) to a calcic extreme (called anorthite). Plagioclase composition is generally expressed by the molecular percentage of anorthite 
(An), and they are traditionally referred to the names albite (0-10 mol\% An), oligoclase (10-30 mol\% An), andesine (30-50 mol\% An), labradorite (50-70 mol\% An), bytownite (70-90 mol\% An) and anorthite (90-100 mol\% An). Plagioclases may also contain certain amount of the potassic extreme (the third point which completes the feldspars triangle, called orthoclase). In the compositional range from labradorite to anorthite, this content is lower than $5 \mathrm{~mol} \%$ and it gradually increases with the albite $(\mathrm{Ab})$ content.

Plagioclase crystals are generally zoned, i.e., they present a concentric structure with narrow bands of different composition. This structure can be readily observed in thin sections with an optical microscope. The anorthite content normally varies between 1 and $30 \%$ within a zoned plagioclase crystal [12]. It is possible to infer the history of the magmatic system by studying the compositional changes along the crystals in a plagioclase, since the composition of a magmatic mineral depends on the temperature, pressure, fluids content and composition of the magma [13, 14].

The study of zoning in plagioclase crystals has been addressed through different techniques. The simplest method consists in producing a concentration profile by electron microprobe point analysis. Optical and Nomarski differential interference contrast microscopy [15] are widely used to obtain high resolution images of zoning, but they provide no quantitative information about chemical composition. Laser interference microscopy has been used in combination with electron probe microanalysis for quantitative profiles of anorthite [16], although the spatial resolution of the former is poor.

Backscattered electron images are a promising alternative to study plagioclase zonations $[12,17,18]$, since they are highly sensitive to small changes in mean atomic number $\bar{Z}$. Nevertheless, these images do not straightforwardly provide quantitative information about the chemical composition, and they must be therefore calibrated in order to obtain the anorthite content. Taking into account that the mean atomic numbers of $\mathrm{Ab}$ and $\mathrm{An}$ are 10.77 and 11.85 , respectively, it can be concluded that $\bar{Z}$ and thus the BE signal increase with the An content in a plagioclase. Due to the small variation of $\bar{Z}$ from $\mathrm{Ab}$ to An, the calibration between the gray levels and the plagioclase composition can be performed by a linear function. Ginibre et al. [12] calibrated the BE images with point quantitative characteristic X-ray analyses performed along a line perpendicular to the zonation edges. Triebold et al. [19] proposed a similar method with quantitative analysis in a complete line scan. Both authors related the An concentration with the $\mathrm{Ca}$ and $\mathrm{Na}$ elemental concentration determined by the X-ray microprobe analysis. 
Although the methods mentioned above had been applied several times, they present some experimental and processing difficulties. For instance, the quantifications are performed along a unique profile and then the calibration is used to extend the quantitative characterization to the entire image. The profile must therefore be representative of the entire BE image. In addition, the interfaces between regions with different $\bar{Z}$ are responsible for a contrast intensification in the BE image (see below) and the quantitative analyses performed at these points will not follow the general tendency in the calibration curve. This fact could be an important source of error when a few points are used for calibrating the BE image. On the other hand, if the measuring equipment does not have an automated X-ray spectra acquisition routine, the number of points to analyze must be significantly reduced in order to optimize the measuring time.

In this work, the calibration of BE images is accomplished through a new approach, in which an anorthite concentration map is obtained from $\mathrm{Ca}$ and Na X-ray maps; the BE image size is reduced to the An map size and a linear calibration between the gray level in the reduced $\mathrm{BE}$ image and the An map is performed. Finally, the linear calibration is applied to the original BE image, obtaining a high resolution An distribution map. With this approach, fluctuations of different origin are smoothed, and the uncertainties in the results are therefore reduced, as shown below. In addition, the fraction of points with contrast enhancement due to interface crossing is greatly reduced, since the calibration is performed with high number of points $\left(\approx 10^{4}\right)$. Monte Carlo simulations were performed to study the resolution of $\mathrm{BE}$ and $\mathrm{X}$-ray signals as a function of the plagioclase composition and the incident electron beam energy. This study permitted to choose the best experimental conditions to optimize the spatial resolution. The methodology was tested in plagioclases from a lava sample from the Cerro Vilama, a quaternary volcano located in the Central Andes, on the international border between Argentina and Bolivia.

The method proposed here is applicable to any material sample in which the mean atomic number contrast depends on the variation of a single component, maybe present as solid solution or separately. This is a common situation in many alloys [20] or mineral series different from those considered here. 


\section{Materials and methods}

Plagioclases from a thin section were studied through the methodology proposed here. To this aim, $\mathrm{Ca}$ and $\mathrm{Na}$ X-ray maps have been acquired in order to perform the BE image gray level calibration with An content, as described below. ${ }^{* * *}$ Henceforth, all concentrations will be given as wt.\%.***

\subsection{X-ray maps and BE image calibration}

In conventional electron probe quantitation, the characteristic intensity $I_{j}$ emitted by each element of an unknown sample is recorded and then compared with the corresponding intensity $I_{j}^{o}$ emitted from a standard of concentration $C_{j}^{o}$. As a first approximation, the intensity $I_{j}$ may be taken as proportional to the mass concentration $C_{j}$ of element $j$ :

$$
\frac{I_{j}}{I_{j}^{o}} \approx \frac{C_{j}}{C_{j}^{o}}
$$

The comparison with standards cancels out geometrical and physical factors which are difficult to determine [21]. The previous relation has no rigorous validity, and matrix effects must be included to produce a more accurate relation. These effects are usually referred to as " $Z A F$ correction" $[1,21]$ : $Z$ and $A$ factors represent the generation, scattering and absorption effects, whereas the $F$ factor involves secondary fluorescence enhancement. The magnitudes of $Z, A$, and $F$ strongly depend on the experimental conditions, mainly on the incident beam energy, X-ray take-off angle and differences in the composition of the standards and the sample. In some cases, it is possible to reduce their influence and even to compensate effects, provided that the experimental conditions are adequately chosen. The composition of the standard used in this work (a homogeneous plagioclase with 65\%. An from SPI mineral standards) is similar to the sample studied, thus, in the energy and concentration ranges involved in this study the $Z A F$ corrections can be considered as a constant for each element (see section 4).

The ratio between $\mathrm{Ca}$ and $\mathrm{Na}$ concentrations for pixel $(i, j)$ can then be written as

$$
\frac{C_{\mathrm{Ca}}(i, j)}{C_{\mathrm{Na}}(i, j)}=\frac{C_{\mathrm{Ca}}^{o}}{C_{\mathrm{Na}}^{o}} \frac{I_{\mathrm{Ca}}(i, j)}{I_{\mathrm{Na}}(i, j)} \frac{I_{\mathrm{Na}}^{o}}{I_{\mathrm{Ca}}^{o}} \frac{(Z A F)_{\mathrm{Na}}}{(Z A F)_{\mathrm{Ca}}},
$$

where $C_{\mathrm{Ca}, \mathrm{Na}}, I_{\mathrm{Na}, \mathrm{Ca}}$ and $Z A F_{\mathrm{Na}, \mathrm{Ca}}$ are, respectively, the concentrations, integrated intensities and $Z A F$ corrections for the elements considered, and the superscript $O$ refers to the standard. The $Z A F$ correction values were 
estimated by performing quantitative point analyses. The anorthite content $A n(i, j)$ (wt.\%) for the pixel $(i, j)$ can be related to the $\mathrm{Ca}$ and $\mathrm{Na}$ wt.\% using the stoichiometric formulae for An and $\mathrm{Ab}$ minerals

$$
\begin{gathered}
C_{\mathrm{Ca}}(i, j)=14.41 A n(i, j), \\
C_{\mathrm{Na}}(i, j)=8.77[100-A n(i, j)],
\end{gathered}
$$

where 8.77 and 14.41 are the concentrations (wt.\%) of $\mathrm{Na}$ in pure albite and $\mathrm{Ca}$ in pure anorthite. From the ratio $C_{\mathrm{Ca}} / C_{\mathrm{Na}}$ determined by Eq. (1), the anorthite content $A n(i, j)$ for each pixel $(i, j)$ can therefore be obtained from these relations

$$
A n(i, j)=8.77 \times \frac{\frac{C_{\mathrm{Ca}}}{C_{\mathrm{Na}}}(i, j)}{14.41+8.77 \times \frac{C_{\mathrm{Ca}}}{C_{\mathrm{Na}}}(i, j)} \times 100 .
$$

In order to assess Eqs. (1) and (2) for each pixel of the XRMs and therefore construct a map of the anorthite content, a routine in Matlab ${ }^{\circledR}$ environment was developed. It is important to notice that the size of the An map obtained is equal to the size of the XRMs, and its spatial resolution is limited by the volume from which the characteristic X-rays emerge from the sample.

As mentioned in the Introduction, it is possible to perform a linear calibration between the BE image gray level and the corresponding anorthite content for each sample point. With the aim of establishing a pixel-to-pixel correspondence, the BE image size was reduced to the An map size. The value for each pixel in the reduced BE image is assessed as the average within a sub-array surrounding the corresponding pixel in the original BE image, covering all the scanned area and avoiding overlaps. An important advantage of this approach is the high number of points available for performing the calibration, which leads to better results since the statistical fluctuations and the relative number of points presenting the BE contrast enhancement due to interface crossing (see section 4.2) is reduced.

\subsection{Monte Carlo simulations}

In order to investigate the interaction volume for BE and characteristic $\mathrm{X}$-ray signals as a function of the incident electron energy and the plagioclase composition, a series of Monte Carlo simulations were performed. The spatial 
resolution is related to this interaction volume, which limits the sensitivity to detect compositional variations in the BE images and X-ray maps.

The example main program PENCYL distributed with the 2008 version of the PENELOPE routine package [22] was used for the simulations. This program is optimized for cylindrical geometries constituted by different layers. The output provided by the program for the X-ray emission is integrated over all the possible take-off directions (ranging from $0^{\circ}$ to $90^{\circ}$ ). Taking into account that the absorption of photons in the sample strongly depends on the take-off angle [5] and that the geometry is well defined in the experiment, the results of the simulations performed with this program are not compatible with the measurements. For this reason, the PENCYL program was modified to register only the photons emitted within directions close to the experimental take-off angle. In those cases in which the symmetry characterizing the normal-incidence EPMA experiment for flat homogeneous samples, all the azimuthal angles were integrated in order to reduce statistical uncertainties for the computing times used. An additional modification to PENCYL was introduced in order to register the radial coordinate of the backscattered electrons when they emerge from the sample. As will be shown below, this radial coordinate is useful to determine the spatial resolution of the BE signal.

A first set of Monte Carlo simulations were performed for a homogeneous material composed by $60 \%$. An for electron incident energies ranging from 5 and $15 \mathrm{keV}$ in order to obtain the ultimate resolution of the BE and characteristic X-ray signals.

As mentioned above, near the interface between materials with different mean atomic numbers, a contrast exaggeration in the BE signal is produced. In order to investigate this effect, a second set of Monte Carlo simulations was performed for samples with an interface separating two materials A $\mid \mathrm{B}$, as shown in Fig. 1. To adequately represent this type of interfaces keeping the cylindrical symmetry imposed by the program PENCYL, the materials $\mathrm{A}$ and $\mathrm{B}$ were associated to concentric cylinders with radius at the interface large enough to ensure that the flat interface approximation was valid at a micrometer scale.

In order to represent a zoning in a plagioclase, three samples were simulated: the material B was fixed as pure anorthite (An 100\%), and three different An wt. concentrations $(60 \%, 70 \%$ and $80 \%)$ were set as the material A. Three incident energies $E_{o}$ were simulated, i.e., 5, 10 and $15 \mathrm{keV}$. For each interface and incident energy, the incident beam impact point $x_{i}$ 


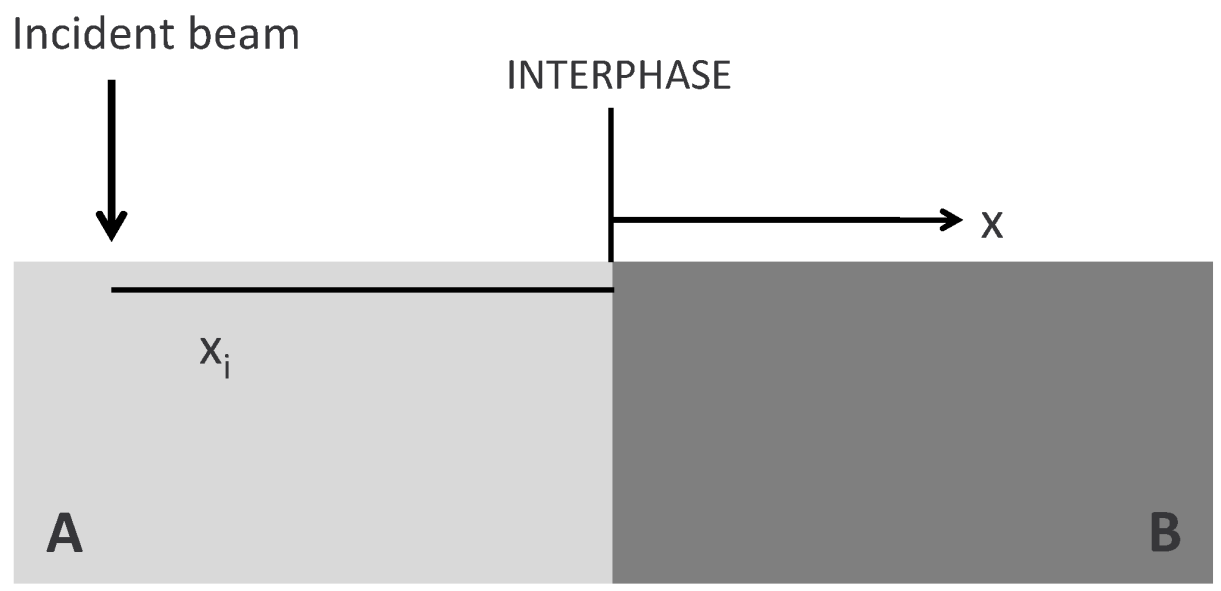

Figure 1: Settings used to study the spatial resolution for sodium and calcium characteristic X-rays, as well as the interface crossing effect in the BE signal near to the interface A $\mid$ B defined by the A (left) and B (right) materials.

was varied from $-7 \mu \mathrm{m}$ to $7 \mu \mathrm{m}$ in steps of $0.25 \mu \mathrm{m}$ (see Fig. 1). At each $x_{i}$ point, the radial distribution of the backscattered electrons and characteristics X-rays corresponding to $\mathrm{Na}$ and $\mathrm{Ca}$ were recorded. Other interfaces A | B defined by materials with very different mean atomic number, such as $\mathrm{Mg} \mid \mathrm{Cu}$ and $\mathrm{C} \mid \mathrm{Pb}$ were simulated to study the behavior of the BE signal exaggeration with the chemical composition.

The simulations were run in a cluster facility at the Facultad de Matemática, Astronomía y Física of the Universidad Nacional de Córdoba, with 3.0GHz Pentium IV hyperthreading processors. In the characteristic X-ray simulations carried out, the incident electron trajectories were surveyed down to certain threshold energy, since no further ionization is possible under this value. This criterion permits to obtain results with appropriate statistical uncertainties in reasonable CPU times, avoiding the computation of events out of interest. With this restriction, relative errors below $1 \%$ for all characteristic X-rays were obtained. For the BE signal simulations, electron energies were considered down to $100 \mathrm{eV}$. This lower limit adequately reproduces the actual experimental BE detection [1]. 


\section{Experimental}

The thin slice of the Cerro Vilama rock was prepared following the standard procedure for this type of material. The sample was coated with a carbon layer $30 \mathrm{~nm}$ thick in order to avoid charging effects. The BE images, XRMs and point X-ray emission spectra were acquired with an environmental SEM (ESEM FEI Quanta 200) furnished with a silicon drift X-ray detector. The XRMs were measured with a resolution of $128 \times 100$ pixels, $E_{o}$ of $10 \mathrm{keV}$, beam current of $7 \mathrm{nA}$, acquisition live time of $1.4 \mathrm{~s}$ per pixel and counting rate of around $30000 \mathrm{c} / \mathrm{s}$. The point analyses were carried out under the same conditions, but the acquisition live time was set to $100 \mathrm{~s}$; in this case, the Plagioclase An65 (no. 35) standard from the commercial SPI \#02753-

AB mount was used. For the BE images, $1024 \times 768$ frames were taken at 5 $\mathrm{ms} /$ pixel, choosing an incident energy of $10 \mathrm{keV}$ for the reasons explained in 4.1. In order to clearly show the effect of BE contrast enhancement due to interface crossing, additional BE images were measured on a polished section of another rock having an interface defined by quartz and rutile.

\section{Results and discussion}

\subsection{Spatial resolution of $B E$ and $X$-rays}

The fraction of backscattered electrons has been surveyed through Monte Carlo simulations for a homogeneous An 60\% plagioclase excited at 5, 10 and $15 \mathrm{keV}$. The program developed allows to discriminate the lateral coordinate $x$ of the point where the backscattered electron has been produced. A histogram can be therefore constructed, which allows to visualize the distribution of the backscattered electron yield around the beam impact point, as shown in Fig. 2.

In all cases, there is a narrow peak centered at the impact point $x=0$ mounted on a wider distribution. The central peak accounts for the fraction of electrons that have a few interactions before being backscattered, and its width is similar to the electron mean free path in the considered material [1]. The overall curve can be represented as the sum of a narrow Lorentzian distribution and a wide Gaussian distribution. Bearing this in mind, the full width at half maximum of the Gaussian distribution is a good estimate for the spatial resolution. As can be observed in the bottom plot of Fig. 2, the resolution is better for low incident electron energies and it varies slightly with An concentration. 

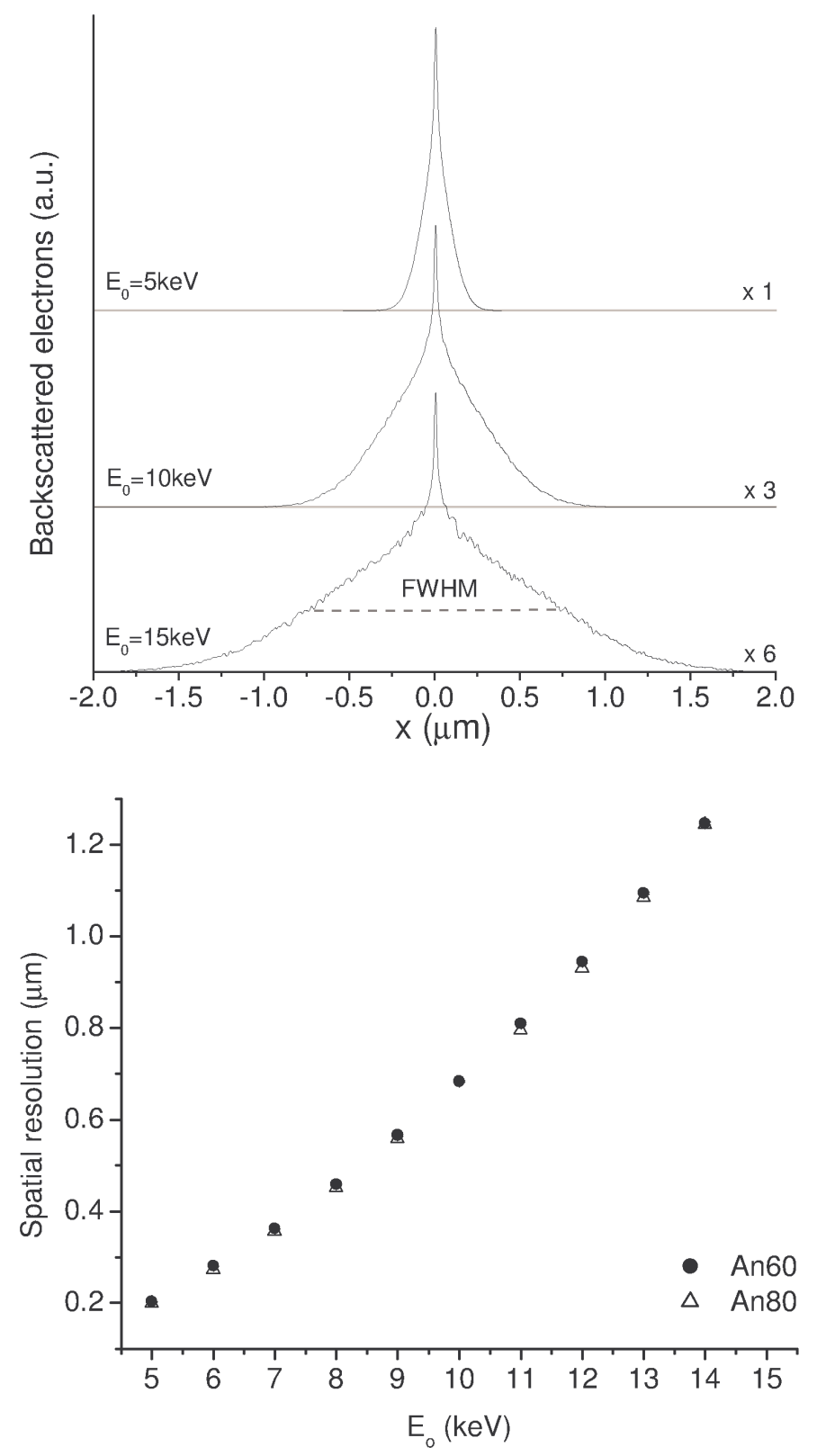

Figure 2: Results of Monte Carlo simulations for the backscattered electron spatial distribution in a labradorite plagioclase (An 60\%) around the electron impact point at 5, 10 and $15 \mathrm{keV}$ (top). Spatial resolution for BE signal in An 60\% and An 80\% (bottom). 

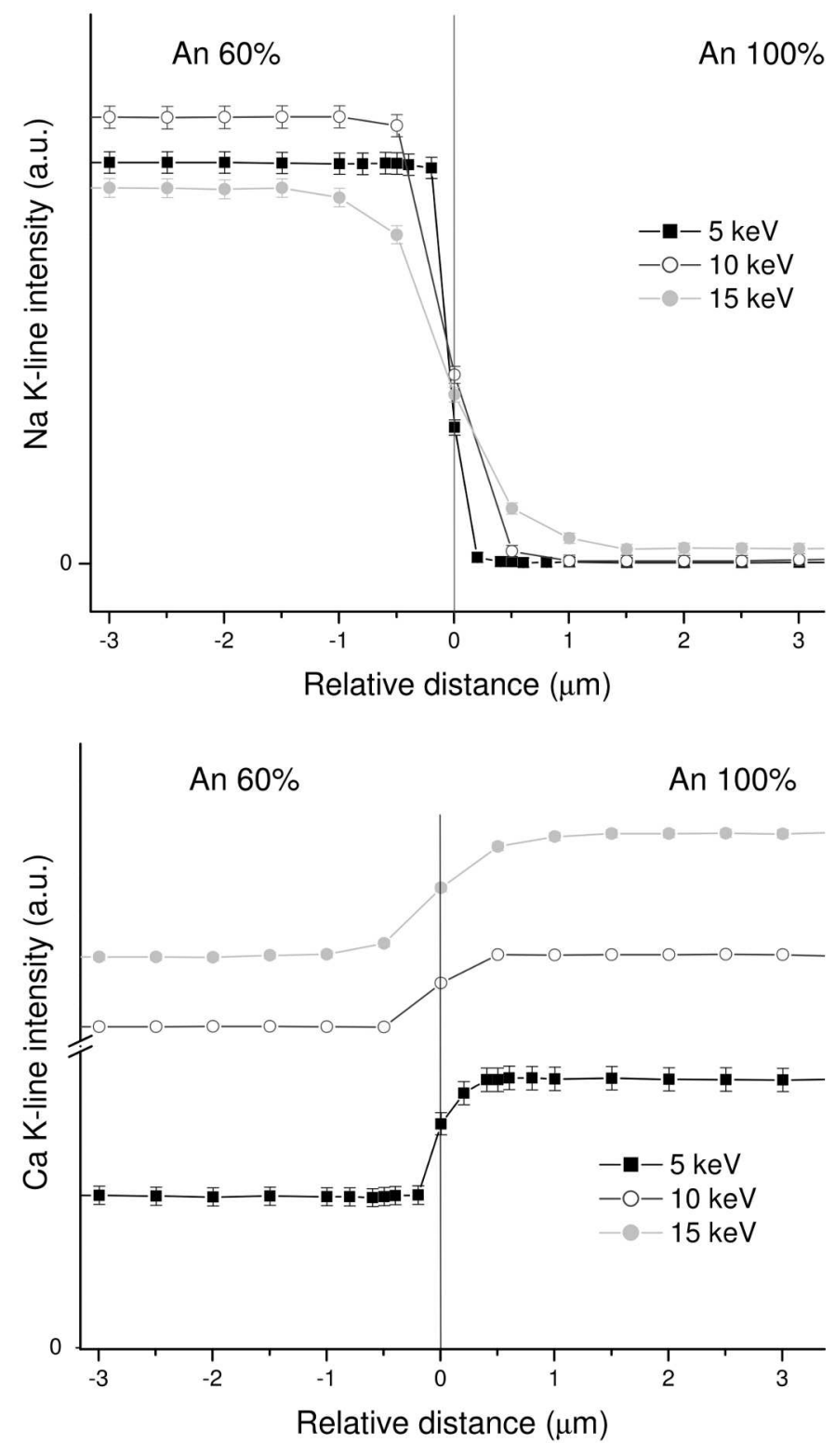

Figure 3: $\mathrm{Na}$ (top) and $\mathrm{Ca}$ (bottom) X-ray intensities for a sample with an interface separating An 60\% | An 100\%, obtained by Monte Carlo simulation for incident energies of 5,10 and $15 \mathrm{keV}$. 


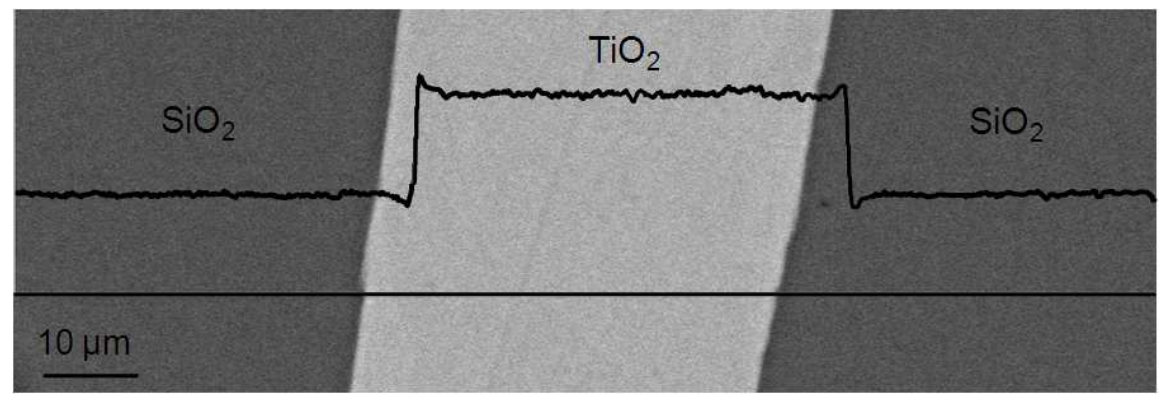

Figure 4: Experimental gray level variation along a profile corresponding to a rutilated quartz sample, measured at $15 \mathrm{keV}$.

Fig. 3 shows the results obtained for the $\mathrm{Na}$ and $\mathrm{Ca}$ characteristic $\mathrm{X}$ ray intensities as a function of the incident beam position $x_{i}$ around an interface separating An 60\% | An 100\%, for incident energies of 5, 10 and $15 \mathrm{keV}$. Although the lateral resolution is commonly defined as the width from $16 \%$ to $84 \%$ of a line scan, it is obvious that when the effect of BE contrast enhancement due to interface crossing is important, this criterion cannot be considered, since the regions involving this contrast intensification would be disregarded. A reasonable estimate for the spatial resolution of the X-ray signal is given by the distance from the interface to the point at which the intensity becomes constant. According to this definition, the spatial resolutions for $\mathrm{Na}$ and $\mathrm{Ca}$ signals result $1 \mu \mathrm{m}$ and $0.5 \mu \mathrm{m}$ at $10 \mathrm{keV}$, respectively. Similarly to the BE signal, the resolution of the X-rays depends on the incident electron energy.

\subsection{Signal distortion in BE images due to interface crossing}

Fig. 4 corresponds to a BE image of a thin section of a geological rutilated quartz. The interfaces $\mathrm{SiO}_{2} \mid \mathrm{TiO}_{2}$ can be considered as "ideal", due to the low solubility of rutile in quartz [23]. As it is shown in the line profiles in this figure, at the vicinity of the interface the $\mathrm{SiO}_{2}\left(\mathrm{TiO}_{2}\right) \mathrm{BE}$ signal decreases (increases) from the average value corresponding the phase.

The Monte Carlo simulations performed for interfaces separating materials with different mean atomic numbers $\bar{Z}$ are useful to explain this behavior. For example, in Fig. 5, the lateral distribution of backscattered electrons at different positions relative to the interface for a sample composed by $\mathrm{Mn} \mid \mathrm{Cu}$ is shown. When the electron beam is impinging the material of lower $\bar{Z}$, the 


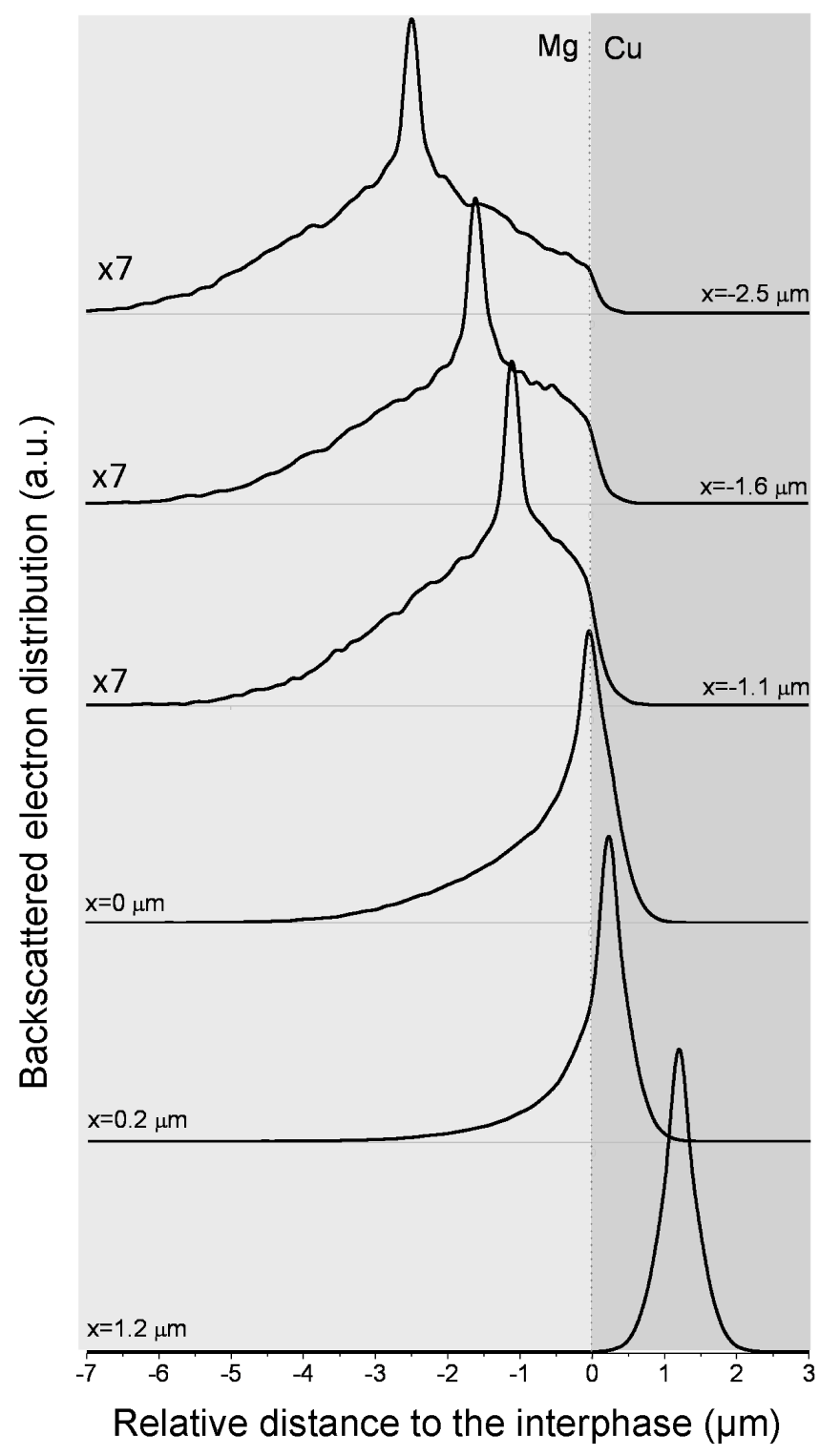

Figure 5: Monte Carlo simulations for the lateral distribution of backscattered electrons at different distances $x_{i}$ from the impact point, for a beam energy of $20 \mathrm{keV}$. The interface corresponds to a $\mathrm{Mg} \mid \mathrm{Cu}$ system. The vertical scales in the first three plots were multiplied by 7 to help visualization. 

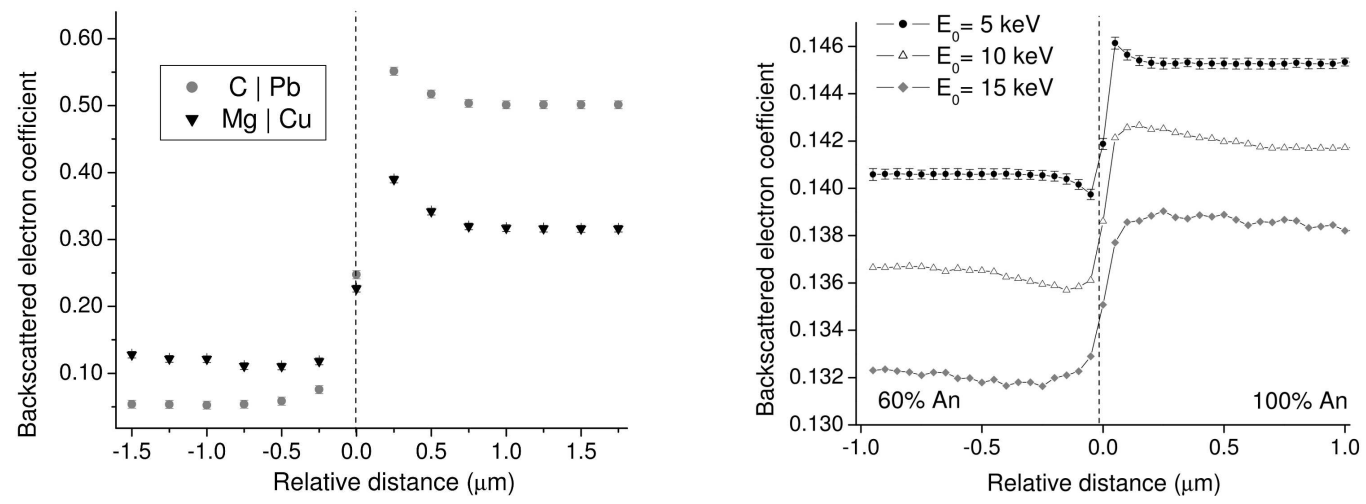

Figure 6: Monte Carlo simulations for the backscattered electron distribution as a function of the distance relative to the interface for $\mathrm{Mg}|\mathrm{Cu}, \mathrm{C}| \mathrm{Pb}$ irradiated at $20 \mathrm{keV}$ (right) and An $60 \%$ An 100\% irradiated at 5, 10, and $15 \mathrm{keV}$ (left).

material with higher $\bar{Z}$ strongly absorbs a portion of the backscattered electrons, and therefore, the BE signal decreases. Analogously, when the impact point is located at the material of higher $\bar{Z}$, the lighter material permits a greater number of backscattered electrons to leave the interface, increasing the BE signal.

Profiles corresponding to the electron backscattering yield for interfaces of $\mathrm{Mg}|\mathrm{Cu}, \mathrm{C}| \mathrm{Pb}$ irradiated at $20 \mathrm{keV}$, and for an interface An $60 \% \mid \mathrm{An}$ $100 \%$ excited at different energies are shown in Fig. 6. As can be seen, the BE contrast enhancement can reach up to $30 \%(\mathrm{Mg} \mid \mathrm{Cu})$. On the other hand, the $\mathrm{BE}$ signal increases / decreases when approximating to the interface from the material with higher / lower $\bar{Z}$, and it strongly depends on the $\bar{Z}$ differences between the two materials conforming the interface. For example, for the $\mathrm{C} \mid \mathrm{Pb}$ interface, the BE signal remains constant up to a distance of $1 \mu \mathrm{m}$ to the interface in $\mathrm{C}$ and $0.75 \mu \mathrm{m}$ in $\mathrm{Pb}$. At distances closer to the interface, the signal increases up to $10 \%$ for $\mathrm{Pb}$. In the case of An $60 \%$ | An $100 \%$ excited at $10 \mathrm{keV}$, the $\mathrm{BE}$ signal is stabilized at $0.5 \mu \mathrm{m}$ from the interfaces for both materials. It must be stressed that in these figures the ordinate scale has been expanded in order to bring to evidence small variations.

\subsection{Calibration of anorthite maps}

The results obtained by Monte Carlo simulations allow to choose the most adequate electron incident energy $E_{o}$ for the acquisition of X-ray maps 


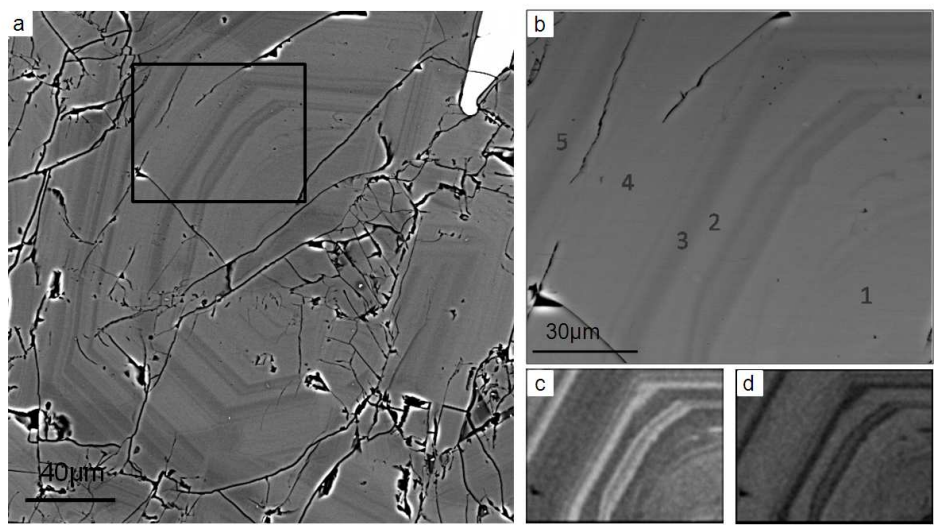

Figure 7: BE image corresponding to crystal 1 (a), and the detailed zone chosen for XRM acquisition (b). Na and Ca XRMs from the selected area (c and d).

Table 1: ZAF corrections for $\mathrm{Ca}$ and $\mathrm{Na}$ in each point analysis and their average value.

\begin{tabular}{lcccccc}
\hline \multicolumn{7}{c}{$Z$ AF CORRECTION } \\
Point & $\mathrm{p} 1$ & $\mathrm{p} 2$ & $\mathrm{p} 3$ & $\mathrm{p} 4$ & $\mathrm{p} 5$ & Average \\
\hline Element & & & & & & \\
$\mathrm{Na}$ & 1.005 & 0.991 & 0.992 & 0.990 & 0.994 & 0.992 \\
$\mathrm{Ca}$ & 1.004 & 0.999 & 0.998 & 0.998 & 0.997 & 0.998 \\
\hline
\end{tabular}

and $\mathrm{BE}$ images. The optimum value turns to be $E_{o}=10 \mathrm{keV}$, since with this energy an efficient $\mathrm{Na}$ and $\mathrm{Ca} \mathrm{K}$ excitation is achieved, maintaining an acceptable spatial resolution. In the case of BE images, three effects must be balanced: for higher incident energies, the chemical contrast is improved, but the contrast enhancement in the zoning edges becomes more evident and the spatial resolution worsens. None of these effects is predominant for an excitation energy of $10 \mathrm{keV}$; for this reason, this incident energy has been chosen for measuring the BE images.

$\mathrm{BE}$ images and $\mathrm{Na}$ and $\mathrm{Ca} \mathrm{X}$-ray maps corresponding to the crystals studied in the plagioclase sample are shown in Figs. 7 and 8. The $Z A F$ corrections obtained for five point analyses and the corresponding averages for $\mathrm{Na}$ and $\mathrm{Ca}$ are shown in table 1 (the selected points are shown in Fig. 7). These $Z A F$ values were obtained by quantitative point analyses in the crystal 


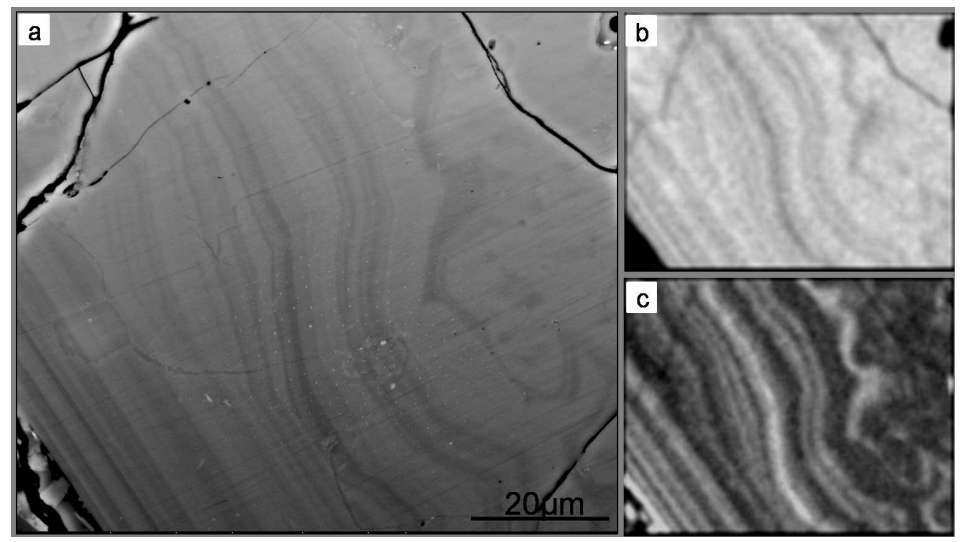

Figure 8: BE image corresponding to crystal 2 (a) and the XRMs for Na and Ca taken in the selected area (b and $c)$.

1 using the equation 1. The selected points are shown in Fig. 7. As can be seen, the variations of the $Z A F$ corrections along the crystal are very small (the maximum difference is $0.9 \%$ ). For this reason, the $Z A F$ corrections were considered as constant for the quantification procedure through the X-ray maps. The An compositional maps for both crystals were therefore obtained from equations 1 and 2 , by using the net X-ray intensity maps of $\mathrm{Ca}$ and $\mathrm{Na}$ and the average $Z A F$ corrections.

In order to perform the calibration between the gray level in the BE image and the anorthite content, it is necessary to make a pixel-to-pixel correspondence. To this aim, the BE images acquired with $1024 \times 768$ pixels were reduced to the size of the anorthite map $(128 \times 100$ pixels $)$. Based on the gray levels corresponding to the minimum and maximum anorthite content, the points associated with fractures or other phases (too light or too dark in the BE image) were discarded in the reduced BE image. The number of points considered as good for the calibration resulted around 9,000 for both crystals.

To obtain the linear calibration, the concentrations associated with the same gray level were averaged. The relation between the gray level and the An concentration for the two crystals is shown in Fig. 9. The error bars correspond to one standard deviation of the concentrations associated with the same gray level. These errors are related to interface effects (see Fig. 4) and to the difference in the spatial resolution of BE and X-ray signals. 

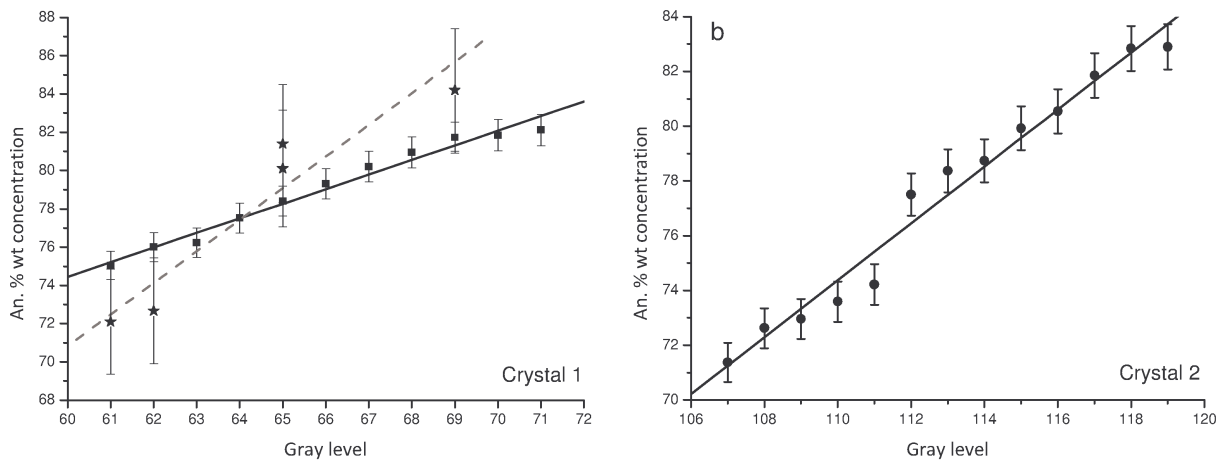

Figure 9: Calibration curves (full line) for the An $\%$ as a function of the BE image gray levels for crystal 1 (left) and crystal 2 (right). The dashed curve corresponds to the fitting to the five conventional point analyses performed.
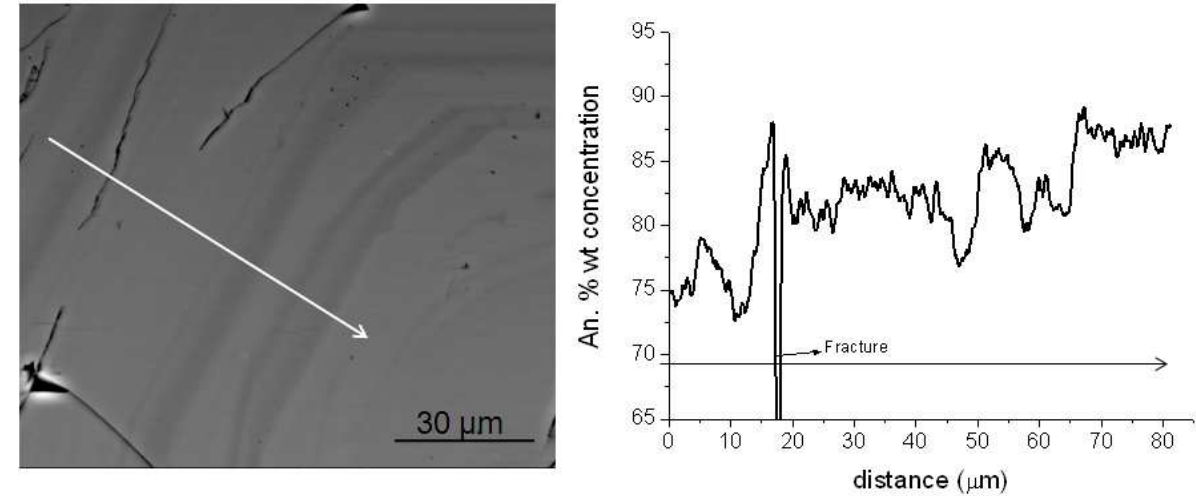

Figure 10: Calibrated BE images (right) and quantitative An-calibrated gray-level profile in the indicated zones for the crystal 1.

The An concentrations obtained from the five point analyses performed at crystal 1 are also included in Fig. 9. As can be observed, the deviation of these point analyses from the linear trend is higher than the errors associated to the present method. It must be emphasized that the individual quantitative analyses are highly influenced by the interface proximity at each point, raising the corresponding uncertainties up to 50\%; obviously the linear fit produced with this procedure is rather different from the one proposed here. It is quite clear that the reduction of uncertainties mentioned above allows to obtain a 


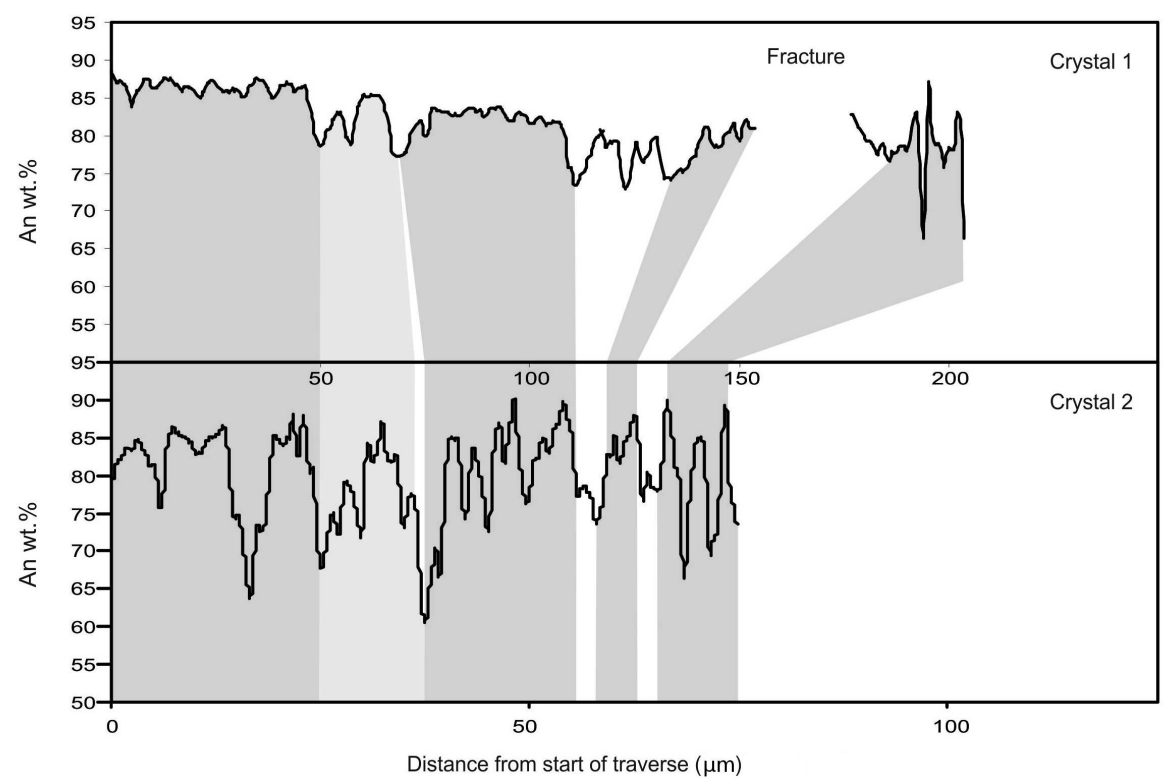

Figure 11: An-calibrated gray-level profiles from the crystals studied. The gray zones indicate the possible correlation between regions with similar oscillation patterns and composition on both crystals from the same rock.

more reliable calibration with the approach presented here. The coefficients of the linear fit were used to transform the original BE image into a high resolution anorthite map (see Fig. 10).

\subsection{Petrological observations on the studied plagioclase crystals}

Although a petrological study of these results is out of the scope of this paper, some concepts about the images and compositional data obtained in this work can be highlighted here. Crystal 1 (Fig. 7) shows zonation of the oscillatory type, with four sharp drops in the An content (indicated by the rise in the gray level). Crystal 2 (Fig. 8) also shows oscillatory zoning; however, its principal characteristic is the presence of a conspicuous resorption surface. This surface surrounds a sieve-textured and irregular core (probably distorted by dissolution), which served as a nucleus for the subsequent growing of the oscillatory-zoned sector. When the images and compositional profiles from the two crystals are compared, bands with equivalent zoning patterns separated by correlated An drops are identified on both (Fig. 11). 
These common bands and An drops are explained by a similar history of growing inside the magma, under the same conditions of temperature, pressure, fluids pressure and melt composition. The oscillatory patterns here observed may be compared with the saw-tooth with resorption, low-amplitude oscillation and high-frequency, high-amplitude oscillation patterns described in [12]. Moreover, the origin of the saw-tooth with resorption pattern in our rocks may be correlated to turbulent convection inside the magma, as they hypothesize in their work. Further work on the cerro Vilama lavas may be necessary to give an accurate description of the history of this magmatic system.

Previous studies in other plagioclase crystals of the same thin slice achieved by profiling through electron microprobe point analysis indicated a small An compositional range, from $79 \%$ to $89 \%$ [24]. The new methodology revealed a compositional range from $66.4 \%$ to $88.7 \%$ in crystal 1 and from $60.5 \%$ to $90.2 \%$ in crystal 2 . Compared to the previous study, the new compositional spectrum is enlarged towards more evolved compositions. This may be due to the incorporation of thin labradorite zones $(<70 \mathrm{~mol} \% \mathrm{An})$ that had been hidden to the eye-guided profiling with electron microprobe. On these grounds the new methodology becomes an ideal tool for the petrologist, because it permits to analyze compositions with a resolution as high as 0.5 $\mu \mathrm{m}$ and, maybe more important, allows to observe the whole crystal on a BE mode and to choose where to perform the profile.

\section{Conclusions}

A methodology for the attainment of high resolution quantitative maps of anorthite concentration in single plagioclase crystals has been developed. Besides its low cost, the method presented in this work is rapid and reliable to obtain bidimensional analyses, which are helpful to distinguish characteristics of the internal morphology of the plagioclase crystals, particularly resorption surfaces and growing zones. The combined use of BE images and their associated compositional maps and profiles results in a very useful tool to analyse oscillatory patterns, providing a direct way to study the magma chamber dynamics and petrological evolution. The methodology was tested on volcanic rocks with basic plagioclase, and it may be suitable for more evolved plagioclases as well as for the study of plutonic rocks.

The procedure proposed for calibrating BE images allowed to obtain quantitative maps of plagioclase composition with the resolution associated 
to the BE image. An important advantage of this approach is the high number of points available for performing the calibration, which leads to more accurate results, since the statistical fluctuations and the relative number of points presenting the BE contrast enhancement effect due to interface crossing is reduced.

Monte Carlo simulations allowed to determine the ultimate resolution of characteristic X-rays and BE signal for a plagioclase sample. This is the first exhaustive study of the spatial resolution of both signals in a plagioclase sample analyzed with an electron microprobe. Particularly, the modifications introduced in the PENCYL code allowed to obtain the lateral distribution of backscattered electrons as a function of the relative position at an interface, which permitted to explain the contrast enhancement effects near interfaces.

The method proposed here is applicable to any material sample in which the mean atomic number contrast depends on the variation of a single component, maybe present as solid solution or separately. This is a common situation in many alloys [20] or different mineral series. Future work may enable to apply the methodology to other types of binary solid solution minerals, especially to rock-forming minerals such as olivine and orthopyroxene. In the case of ternary systems, where all three components may vary, it is often necessary to analyze variations along isothermal regions, which furnishes an additional condition constraining the system in such a manner it

can again be described in terms of a single varying concentration: again, a calibration will be possible, consisting in finding the relationship between the mean atomic number and the concentration of one only component.

\section{Acknowledgements}

This work was partially supported by the Secretaría de Ciencia y Técnica of the Universidad Nacional de Córdoba. The authors are also grateful to the laboratories of the Universidad Nacional de San Luis (LaBMEM) and the Universidad Nacional de la Plata (SMEByM-LIMF).

[1] J. Goldstein, D. Newbury, P. Echlin, D. Joy, A. Romig, C. Lyman, C. Fiori, E. Lifshin, Scanning Electron Microscopy and X-Ray Microanalysis: A Text for Biologists, Materials Scientists, and Geologists, New York: Plenum Press, 1992.

[2] E. Gatti, P. Rehak, Review of semiconductor drift detectors, Nucl. Instrum. Methods Phys. Res. Sect. A 541 (2005) 47-60. 
[3] U. Gernet, Comparing the $\mathrm{Si}(\mathrm{Li})$-detector and the silicon drift detector (SDD) using EDX in SEM, in: M. Luysberg, K. Tillmann, T. Weirich (Eds.), 14th European Microscopy Congress, 2008, pp. 697-698. Aachen, Germany: Springer Berlin Heidelberg.

[4] L. Reimer, Image formation in low-voltage scanning electron microscopy, Bellingham, Washington: SPIE Optical Engineering Press, 1993.

[5] V. Scott, G. Love, S. Reed, Quantitative Electron-Probe Microanalysis, 2nd. ed., New York: Ellis Horwood Ltd., 1995.

[6] P. Roschger, P. Fratzl, J. Eschberger, K. Klaushofer, Validation of Quantitative Backscattered Electron Imaging for the Measurement of Mineral Density Distribution in Human Bone Biopsies, Bone 23 (1998) 319-326.

[7] K.O. Kjellsen, A. Monsy, K. Isachsen, R.J. Detwiler, Preparation of flatpolished specimens for SEM-backscattered electron imaging and X-ray microanalysis-importance of epoxy impregnation, Cem. Concr. Res. 33 (2003) 611-616.

[8] O. Schalm, K. Janssens, J. Caen, Characterization of the main causes of deterioration of grisaille paint layers in 19th century stained-glass windows by J.B. Capronnier, Spectrochim. Acta Part B 58 (2003) 589607 .

[9] V. Galván Josa, S.R. Bertolino, J.A. Riveros, G. Castellano, Methodology for processing backscattered electron images. Application to Aguada archaeological paints, Micron 40 (2009) 793-799.

[10] C.A. Peters, Accessibilities of reactive minerals in consolidated sedimentary rock: An imaging study of three sandstones, Chem. Geol. 265 (2009) 198-208.

[11] K. Keune, A. van Loon, J.J. Boon, SEM Backscattered-Electron Images of Paint Cross Sections as Information Source for the Presence of the Lead White Pigment and Lead-Related Degradation and Migration Phenomena in Oil Paintings, Microsc. Microanal. 17 (2011) 696-701.

[12] C. Ginibre, A. Kronz, G. Worner, High-resolution quantitative imaging of plagioclase composition using accumulated back-scattered electron 
images: New constraints on oscillatory zoning, Contrib. Mineral. Petrol. 142 (2002) 436-448.

[13] T. Grove, M. Baker, R. Kinzler, Coupled CaAl-NaSi diffusion in plagioclase feldspar: experiments and applications to cooling rate speedometry, Geochim. Cosmochim. Acta 48 (1984) 2113-2121.

[14] S.A. Morse, Cation Diffusion in Plagioclase Feldspar, Science 225 (1984) 504-509.

[15] A.H. Clark, T.H. Pearce, P.L. Roeder, I. Wolfson, Oscillatory zoning and other microstructures in magmatic olivine and augite: Nomarski interference contrast observations on etched polished surfaces, Am. Mineral. 71 (1986) 734-741.

[16] T.H. Pearce, J.K. Rusell, I. Wolfson, Laser-interference and Nomarski interference imaging of zoning profiles in plagioclase phenocrysts from the May 18, 1980 eruption of Mount St. Helens, Washington, Am. Mineral. 72 (1987) 1131-1143.

[17] T.H. Pearce, A.M. Kolisnik, Observations of plagioclase zoning using interference imaging, Earth Sci. Rev. 29 (1990) 9-26.

[18] S.L. Higman, T.H. Pearce, Spatiotemporal dynamics in oscillatory zoned magmatic plagioclase, Geophys. Res. Lett. 20 (1993) 1935-1938.

[19] S. Triebold, A. Kronz, G. Worner, Anorthite-calibrated backscattered electron profiles, trace elements, and growth textures in feldspars from the Teide-Pico Viejo volcanic complex (Tenerife), J. Volcanol. Geotherm. Res. 154 (2006) 117-130.

[20] N. Melnychenko-Koblyuk, V.V. Romaka, L. Romaka, Yu. Stadnyk, Interaction between the components in the $\{\mathrm{Zr}, \mathrm{Hf}\}-\mathrm{Ag}-\mathrm{Sn}$ ternary systems, Chem. Met. Alloys 4 (2011) 234-242.

[21] S. Reed, Electron Probe Microanalysis, 2nd ed.; Cambridge: Cambridge University Press, 1993.

[22] F. Salvat, J.M. Fernández-Varea, J. Sempau, PENELOPE-2008, A Code System for Monte Carlo Simulation of Electron and Photon Transport. Issy-les-Moulineaux, France: OECD Nuclear Energy Agency, 2009. 
[23] Y. Ren, Y. Fei, J. Yang, W. Bai, $\mathrm{SiO}_{2}$ solubility in rutile at high temperature and high pressure, J. Earth Sci. 20 (2009) 274-283.

[24] D. Fracchia, Volcanismo postcolapso de la caldera Vilama, Mioceno superior, Puna argentino-boliviana. Mecanismos eruptivos y petrogénesis (in Spanish), PhD thesis, Universidad Nacional de La Plata (2009). 